\title{
Measurement of thermospheric temperatures using OMTI Fabry-Perot interferometers with 70-mm etalon
}

\author{
Y. Nakamura ${ }^{1,2}$, K. Shiokawa ${ }^{3 *}$, Y. Otsuka 3 , S. Oyama 3 , S. Nozawa ${ }^{3}$, T. Komolmis ${ }^{4}$, S. Komonjida ${ }^{4}$, Dave Neudegg ${ }^{5}$, \\ Colin Yuile ${ }^{5}$, J. Meriwether ${ }^{6}, \mathrm{H}$. Shinagawa ${ }^{7}$ and $\mathrm{H}$. $\mathrm{Jin}^{7}$
}

\begin{abstract}
Fabry-Perot interferometer (FPI) is an instrument that can measure the temperature and wind velocity of the thermosphere through observations of airglow emission at a wavelength of $630.0 \mathrm{~nm}$. The Solar-Terrestrial Environment Laboratory/Institute for Space-Earth Environmental Research, Nagoya University, has recently developed four new ground-based FPIs. One of those FPIs, possessing a large-aperture etalon (diameter: $116 \mathrm{~mm}$ ), was installed in Tromsø (FP01), Norway, in 2009. The other three small FPIs, using 70-mm-diameter etalons, were installed in Thailand (FP02), Indonesia (FP03) and Australia (FP04) in 2010-2011. They use highly sensitive cooled-CCD cameras with $1024 \times 1024$ pixels to obtain interference fringes. However, appropriate temperature has not been obtained from the interference fringes using these new small-aperture FPIs. In the present study we improved the analysis procedure of temperature determination using these FPIs. Each of FPIs measures north, south, east and west directions repeatedly by rotating two mirrors mounted on top of the FPI. We estimated center pixel of laser fringe and airglow fringes for each direction and found significant differences in the center pixel locations (a few pixels) among the measurement directions. These differences are considered to be caused by movement of the scanning mirror on the top of the optics, resulting in mechanical distortion of the optics body. By calculating the fringe center separately for each direction, we could correct these center pixel variations and determine the temperature with random errors of 10-40 K. This new method was employed to the all measurements from four FPIs after 2009 and provided temperatures with reasonably small errors. However, we found that temperatures below $400 \mathrm{~K}$ were obtained associated with weak airglow intensities and concluded using a model calculation that they are due to contamination of $\mathrm{OH}$ line emissions in the upper mesosphere. By defining an appropriate threshold of the fringe peak count, we successfully eliminated these unrealistic temperature values, and the corrected temperature values became comparable to those provided by the MSIS-90E and GAIA models.
\end{abstract}

Keywords: Fabry-Perot interferometer, 630.0 nm, Thermosphere, Temperature, Small etalon

\section{Background}

The Earth's oxygen airglow emission at the 630-nm wavelength has an emission layer covering the altitudes from 200 to $300 \mathrm{~km}$ (Tohmatsu 1973). Observations of aurora and airglow using a high-resolution Fabry-Perot interferometer (FPI) permit the remote measurement of the

\footnotetext{
*Correspondence: shiokawa@nagoya-u.jp

${ }^{3}$ Institute for Space-Earth Environmental Research (ISEE), Nagoya

University, Chikusa-ku, Nagoya 464-8601, Japan

Full list of author information is available at the end of the article
}

wind and the temperature of these regions through measurement of the Doppler shift and Doppler broadening of the weak 630.0-nm emission line shape. Remote sensing of the dynamics of thermospheric neutral particles from ground-based stations is difficult when using other photometric techniques that lack the high-resolution capability of interferometry.

The use of the cooled-CCD detector to the FPI permits highly sensitive imaging measurement of the interference fringe ring pattern (e.g., Biondi et al. 1995). Measurements of thermospheric temperatures using the 
large-aperture (typically, diameter: 100-150 mm) FPI have been performed for individual nights (e.g., Hernandez and Roble 1995; Meriwether et al. 1996, 1997), and as statistical analysis (e.g., Killeen et al. 1995, Meriwether et al. 2011). These FPIs requiring large-aperture etalons to measure weak airglow and auroral emissions become very expensive, and the added expense of the instrumentation means that multi-site observation of the thermosphere winds and temperatures is not normally possible. Recently, small-aperture FPIs using a cooled-CCD detector with high quantum efficiency and image-capturing quality have been developed (e.g., Makela et al. 2009, 2011). Shiokawa et al. (2012) achieved the multi-point observation of the thermosphere using three small-aperture FPIs with an etalon diameter of $70 \mathrm{~mm}$. However, appropriate temperature has not been obtained from the interference fringes using these three new small-aperture FPIs.

In this paper, we report improvement of the temperature measurement using the FPIs reported by Shiokawa et al. (2012). In "Instruments" section, we describe the FPIs used in the present study. In "Data analysis" section we explain the problems found during the temperature analysis, and in "Improvement" section we show the improvements. In "Statistical analysis" section, we show results of statistical analysis of temperatures obtained by these FPIs. In "Discussion" section, we discuss the accuracy of the temperature estimation.

\section{Instruments}

As shown in Fig. 1, the Solar-Terrestrial Environment Laboratory (STEL), which is reorganized into the Institute for Space-Earth Environmental Research (ISEE) on October 1, 2015, in Nagoya University, has five FPI observatories as a part of the network of Optical Mesosphere

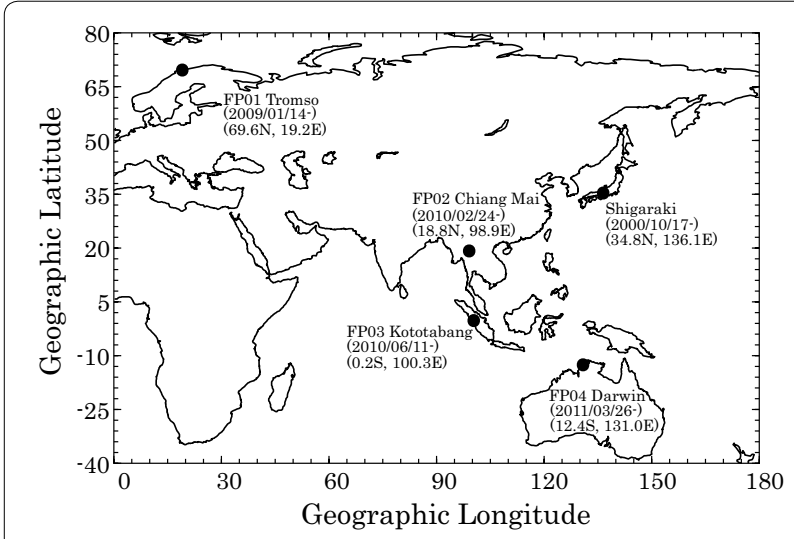

Fig. 1 Site map of the Fabry-Perot interferometers (FPIs)
Thermosphere Imagers (OMTIs) (Shiokawa et al. 1999, 2009). Two (FP00 and FP01) of these FPIs possess a large-aperture etalon with a diameter of $116 \mathrm{~mm}$ and were installed at Shigaraki $\left(34.8^{\circ} \mathrm{N}, 136.1^{\circ} \mathrm{E}\right.$, Shiokawa et al. 2003), Japan on October 17, 2000, and at Tromsø (69.6N, 19.2E, FP01), Norway on January 14, 2009. The other FPIs are equipped with a smaller-aperture etalon having a diameter of $70 \mathrm{~mm}$, and these were installed at Chiang Mai (18.8N, 98.9E, FP02), Thailand, on February 24, 2009, Kototabang (0.2S, 100.3E, FP03), Indonesia, on June 11, 2010, and Darwin (12.4S, 131.0E, FP04), Australia, on March 26, 2011.

Figure 2a, b shows the photograph and the optics of the FP04 at Darwin, Australia. Airglow emissions from the sky are reflected by two $45^{\circ}$ mirrors. Then, the emission line at a wavelength of $630 \mathrm{~nm}$ is extracted by a band-pass filter with a band width of $2.5 \mathrm{~nm}$. Then the emission causes interference in the small etalon, which has a diameter of $70 \mathrm{~mm}$ and a gap of $15 \mathrm{~mm}$. The free spectral range of $15 \mathrm{~mm}$ etalon gap is $\sim 0.013 \mathrm{~nm}$ for the 630-nm airglow. The reflectivities, $\mathrm{R}$, for the etalons of FP01 and FP02-FP04 are 0.85 and 0.76, corresponding finesses of 19.3 and 11.4, respectively. The interference fringe is imaged on the cooled-CCD camera through the focusing lens. The field-of-view and the maximum incident angle of light on the etalon are both $1.4^{\circ}$. The cooled-CCD camera with $1024 \times 1024$ pixels and $13.3 \times 13.3 \mathrm{~mm}$ size was made by Hamamatsu Photonics (C4742-98-26KWG2S) and has a 4-stage thermoelectric cooling system with water circulation, which can cool the $\mathrm{CCD}$ down to $-80{ }^{\circ} \mathrm{C}$. The readout noise and dark noise levels of this CCD camera are 6 electrons (r.m.s.) and 0.0012 electrons/s/pixel, respectively. As explained

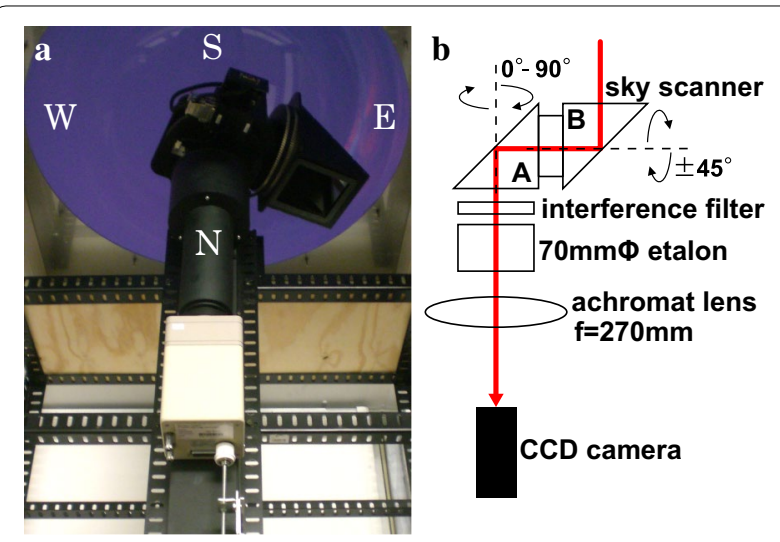

Fig. 2 a Photograph and $\mathbf{b}$ structure of the small-aperture FabryPerot interferometer at Darwin, Australia. Dashed lines and a red line show rotation axes of the sky scanner and a path of an incident light, respectively. The scanners $A$ and $B$ rotate $90^{\circ}$ in azimuthal direction and $\pm 45^{\circ}$ from zenith direction, respectively 
by Shiokawa et al. (2012), all of the FPI optics are calibrated by using a frequency stabilized $\mathrm{He}-\mathrm{Ne}$ laser (Spectra Physics 117A-LW) at a wavelength of $632.8 \mathrm{~nm}$ every night automatically. The laser light is used to determine the instrumental broadening function which is used to estimate the temperature of the emitting species (e.g., Killeen and Hays 1984). Using a multi-mode fiber optic cable, the laser light is passed into a square-shape scattering chamber whose interior walls are painted by white scattering Teflon with an opal glass on the top; this approach achieves a quasi-Lambertian surface. The sky scanner is programmed to look into the opal glass of the calibration laser source by moving to the calibration chamber. FP01 has the same sky scanner and cooledCCD camera with those of the small-aperture FPIs. However, FP01 has a filter wheel to observe three wavelengths (557.7, 630.0 and $732.0 \mathrm{~nm}$ ), four lenses and a large-aperture etalon (diameter: $116 \mathrm{~mm}$ ). The body structure of FP01 is thus quite different from that of other small-aperture FPIs. The field-of-view and the maximum incident angle are $1.3^{\circ}$ and $2.5^{\circ}$, respectively. The details of these FPIs have been shown by Shiokawa et al. (2012).

Figure 3 shows examples of the interference fringes obtained by FP02 for (a) $630.0 \mathrm{~nm}$ (sky) and (b) $632.8 \mathrm{~nm}$ (laser) on December 24, 2010 at Chiang Mai, Thailand. The exposure times are 210 and $0.2 \mathrm{~s}$, respectively. The number of the fringes is 14 . In this study, we use ten fringes from the center of the interference fringe for temperature estimation. The ghost fringes are because of low resolution of the plots and do not exist in the original $1024 \times 1024$ images.

\section{Data analysis}

In this section we introduce the method of temperature estimation from the interference fringes obtained by FPIs. Figure 4 shows a horizontal cross section of onenight average fringes obtained by FP02 for $630.0 \mathrm{~nm}$ on December 24, 2010, at Chiang Mai, Thailand. The crosses indicate the 20 peak locations (ten pairs) identified for each fringe, determined by fitting a Gaussian function. We determine the horizontal and vertical center locations of the circular fringes in pixels by averaging these 20 peak positions of the horizontal and vertical cross sections. One-night average fringe is used to determine the airglow fringe center to reduce the noise due to lowintensity airglow. The same procedure is applied for the laser fringes.

Figure 5 shows examples of fringes (thin lines) integrated over all azimuthal angles from the concentric fringe circle obtained by FP02 for (a) $630.0 \mathrm{~nm}$ (sky) and (b) $632.8 \mathrm{~nm}$ (laser) on December 24, 2010, at Chiang Mai, Thailand. Horizontal axis is in a linear scale for the $r^{2}$ space and corresponds to the distance from the center of the interference fringe. Vertical axis shows the count integrated over the area of each bin in the $r^{2}$-space. The bin in $r^{2}$-space is taken to have an equal annular area size

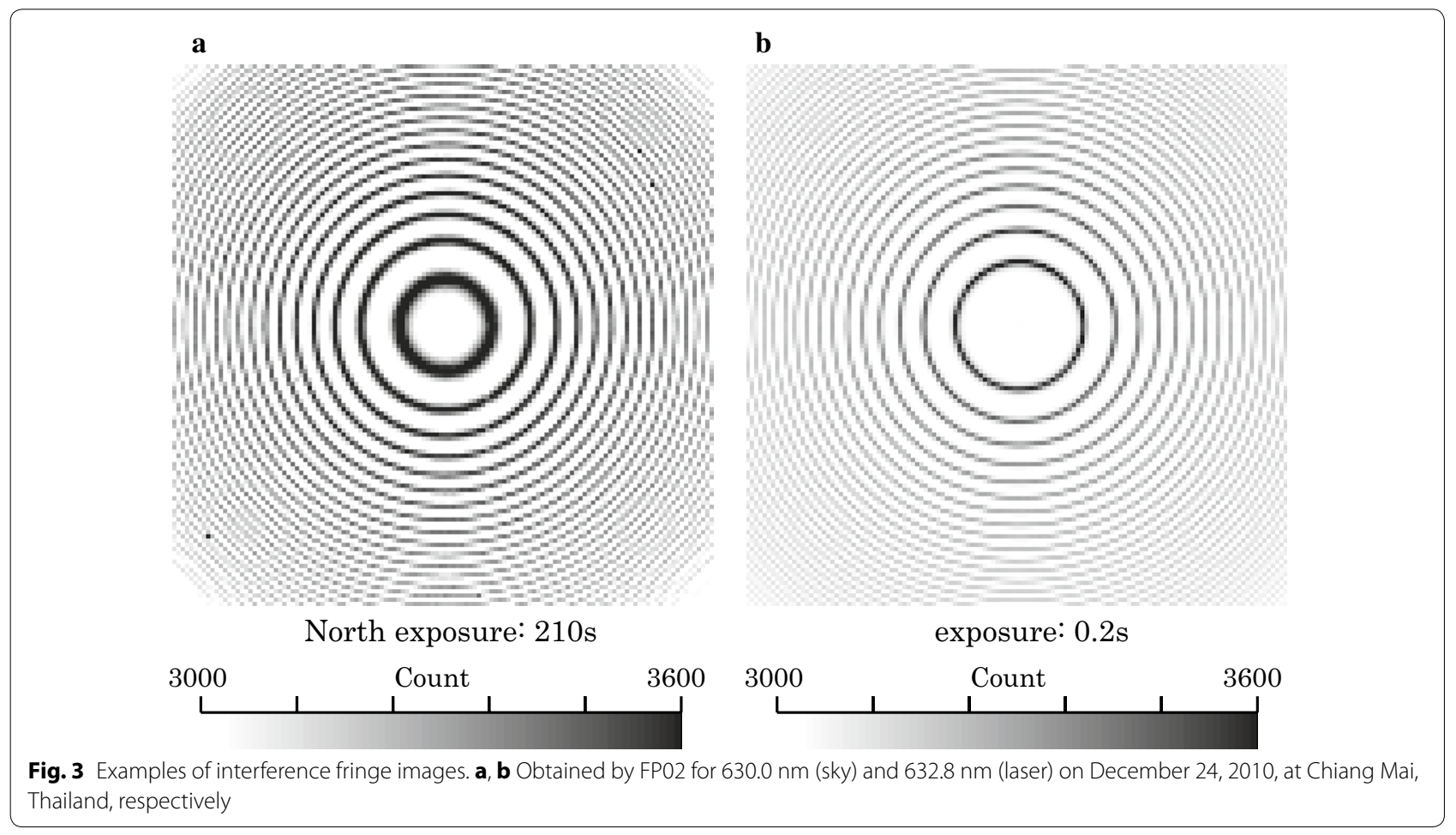




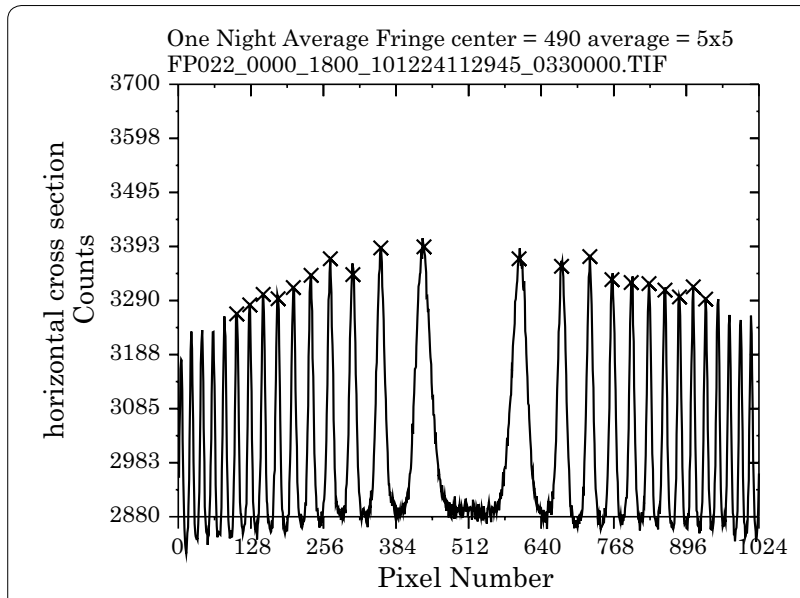

Fig. 4 A cross section of a one-night averaged interference fringe. This was obtained by FP02 for $630.0 \mathrm{~nm}$ on December 24, 2010, at Chiang Mai, Thailand. The crosses indicate the peak locations identified for each fringe that are used to determine the center of the circular fringes
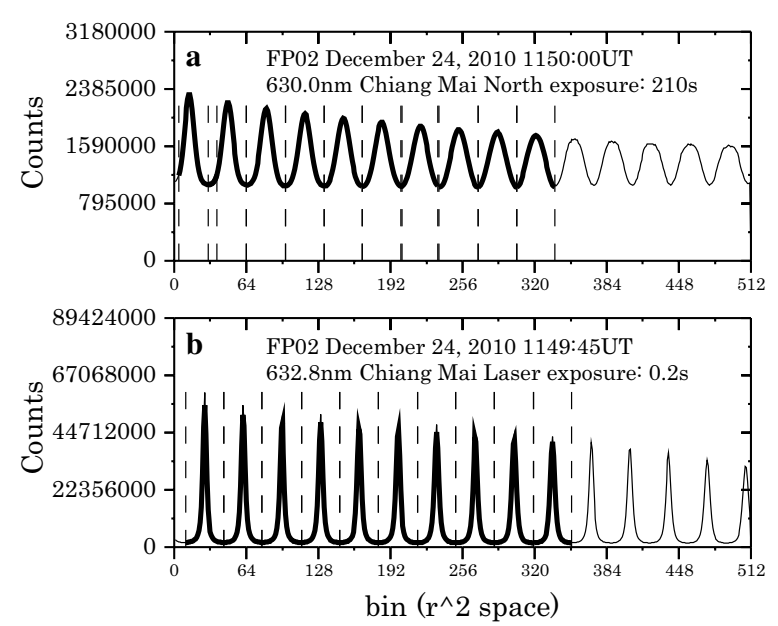

Fig. 5 Example of azimuthally integrated fringes (thin lines). This was obtained by a FP02 for $630.0 \mathrm{~nm}$ (sky) and b FP02 for $632.8 \mathrm{~nm}$ (laser) on December 24, 2010, at Chiang Mai, Thailand. Thick lines indicate the fitting of Eq. (1). The thin vertical dashed lines indicate the intervals of the free spectral ranges used for the fitting

in the original CCD pixel space. The thick line indicates the fitted theoretical curves to estimate the temperature. This theoretical curve is given by Eq. (1) .

According to the Fourier expansion form of FPI interference fringes by Killeen and Hays (1984), the count $N_{i}$ for the $i$ th pixel is given as:

$$
N_{i}=C_{o i} t\left[\left.\frac{\partial R}{\partial \lambda}\right|_{0} \overline{\Delta \lambda_{F}} a_{0}+R_{0} T_{F_{0}}\left(\lambda_{l}\right)\left\{a_{0}+\sum_{n=1}^{m}\left[a_{n} \cos (2 \pi n x)+b_{n} \sin (2 \pi n x)\right] \exp \left(-n^{2} G^{2}\right)\right\}\right]+B_{i} .
$$




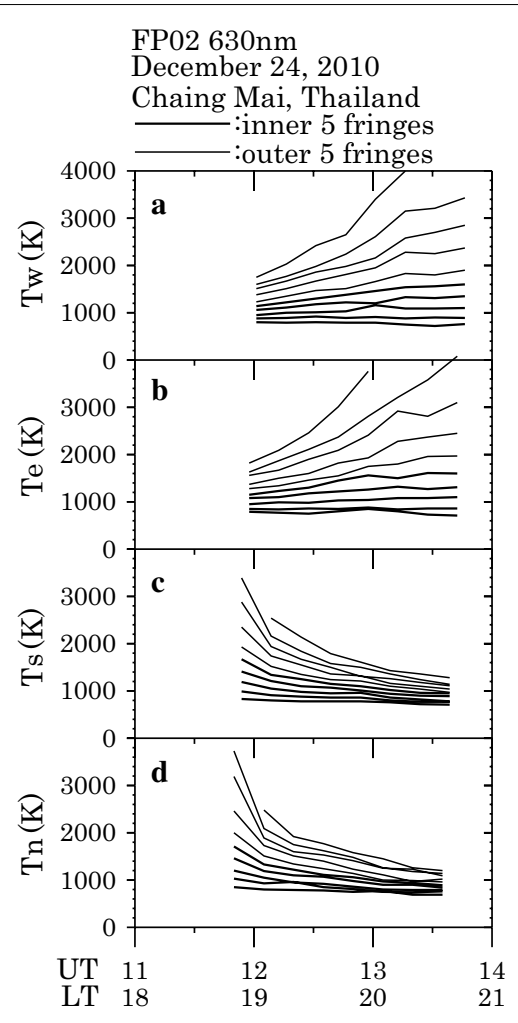

Fig. 6 Thermospheric temperatures for $\mathbf{a}$ west, $\mathbf{b}$ east, $\mathbf{c}$ south and d north directions. This was measured by FP02 for 630.0-nm airglow at Chiang Mai on December 24, 2010

3 problems. First, there are large differences of temperatures over a few hundred Kelvin for different fringes. Second, the temporal variations of temperatures for eastwest and north-south directions show opposite sense. Third, unrealistically high temperatures over $3000 \mathrm{~K}$ were obtained. Similar problems were also identified for FP03 and FP04. Actually we can notice widening of the fringe width toward the outer fringes in the fringe profile shown in Fig. 5a.

\section{Improvement}

The accuracy of the temperature determination strongly depends on the accuracy of the center determination of the interference fringes, because temperatures obtained by FPIs correspond to the width of the fringes. Small FPIs scan the sky in north, south, east, and west and laser is the filter integral width- $\mathrm{A} 90^{\circ}$ and rotating mirror- $\mathrm{B} \pm 45^{\circ}$ or $180^{\circ}$ on the top of the optics.

Table 1 shows differences (in pixel) of the center of interference fringes in all directions obtained by FP01-FP04 for 3 days. "N," "S," "E," "W" and "L" denote the fringe center of north, south, east, west, and laser, respectively. "NS" and "EW" denote the average of the fringe centers of north and south, and east and west, respectively. Differences of the center are shown as "(vertical difference, horizontal difference)". "Average sigma $(K)$ " shows one-night averages of standard deviations of temperatures obtained by ten independent fringes. Differences of the center of the interference fringes between north and south and between east and west directions are very small. However, differences between averaged centers of north-south and east-west are significantly large in the order of 2-7 pixels. This large difference is considered to be the result of a distortion of the optics due to the scanning head weight in zonal and azimuthal direction. Considering the structure of small-aperture FPIs described in Fig. 2b, the optics body below the sky scanner possibly bends and fringe centers for north-south and east-west directions shift depending on the azimuth direction of the sky scanner.

In the previous fringe analysis, we determined the fringe center using one-night average fringes in all directions. In this study, we newly determined the fringe center separately for each direction and then estimated temperatures. In other words, we calculated one-night average fringe for each direction (north, south, east, and west) separately, and calculate the fringe center location for each direction by the procedures described above. This new method significantly improved the quality of the obtained temperatures, as shown below.

In the FP01, fringe centers for different directions are nearly identical in Table 1 . This is probably because the FP01 body is more rigid than that of small-aperture FPIs because of the difference of the optics body structure, as described in "Instruments" section.

Figure 7 shows an example of azimuthally integrated fringes (thin lines) obtained by FP02 for $630.0 \mathrm{~nm}$ (sky) on December 24, 2010, at Chiang Mai, Thailand after the above correction of the center of the interference fringe. In Fig. 5, peak count of the fringe degraded and the width of the fringe become wider with increasing distance from the fringe center. On the other hand, in Fig. 7, the peak count degradation becomes much smaller, and the fringe widths are mostly identical.

Figure 8 shows the time variation of the temperatures obtained for (a) west, (b) east, (c) south and (d) north directions measured by FP02 for 630.0-nm airglow at Chiang Mai on December 24, 2010 after the correction of the center of the interference fringe. From this figure, variations among temperatures obtained from ten fringes are very small in the order of several tens of $\mathrm{K}$. The differences of the temporal variations of temperatures for east-west and north-south directions have not been seen. As shown in Table 1, averages of standard deviations of temperatures are $16 \mathrm{~K}$ on this night (FP02, December $24,2010)$. The standard deviations are mostly $10-40 \mathrm{~K}$ for other nights and other FPIs. From these results, we 
Table 1 Differences (in pixel) of the center of interference fringes obtained by FP01-FP04 for 3 days

\begin{tabular}{|c|c|c|c|}
\hline & \multicolumn{3}{|l|}{ FP01 } \\
\hline & $2011 / 2 / 1$ & $2011 / 12 / 21$ & $2012 / 11 / 7$ \\
\hline$|N-S|$ & $(0.04,0.02)$ & $(0.07,0.00)$ & $(0.05,0.00)$ \\
\hline$|E-W|$ & $(0.06,0.00)$ & $(0.01,0.00)$ & $(0.00,0.01)$ \\
\hline$|N S-E W|$ & $(0.01,0.03)$ & $(0.01,0.00)$ & $(0.02,0.00)$ \\
\hline$|\mathrm{L}-\mathrm{NS}|$ & $(0.21,0.90)$ & $(0.25,0.95)$ & $(0.19,1.29)$ \\
\hline$|L-E W|$ & $(0.22,0.87)$ & $(0.23,0.95)$ & $(0.23,1.39)$ \\
\hline \multirow[t]{3}{*}{ Average sigma (K) } & 26.34018 & 28.69704 & 27.21892 \\
\hline & \multicolumn{3}{|l|}{ FP02 } \\
\hline & $2010 / 12 / 23$ & $2010 / 12 / 24$ & $2012 / 9 / 24$ \\
\hline$|N-S|$ & $(0.67,0.09)$ & $(0.05,0.16)$ & $(0.07,0.05)$ \\
\hline$|E-W|$ & $(0.03,0.09)$ & $(0.05,0.14)$ & $(0.09,0.07)$ \\
\hline$|N S-E W|$ & $(2.63,6.41)$ & $(2.38,6.36)$ & $(2.33,6.20)$ \\
\hline$|\mathrm{L}-\mathrm{NS}|$ & $(0.35,1.00)$ & $(0.07,1.13)$ & $(0.30,0.36)$ \\
\hline$|L-E W|$ & $(2.28,7.41)$ & $(2.45,7.49)$ & $(2.63,6.55)$ \\
\hline \multirow[t]{3}{*}{ Average sigma (K) } & 17.37568 & 16.06653 & 18.1809 \\
\hline & \multicolumn{3}{|l|}{ FP03 } \\
\hline & $2010 / 10 / 16$ & $2011 / 2 / 9$ & $2011 / 4 / 6$ \\
\hline$|N-S|$ & $(0.15,0.25)$ & $(0.01,0.02)$ & $(0.07,0.11)$ \\
\hline$|E-W|$ & $(0.12,0.02)$ & $(0.06,0.05)$ & $(0.06,0.49)$ \\
\hline$|N S-E W|$ & $(0.42,1.32)$ & $(0.31,1.25)$ & $(0.32,1.81)$ \\
\hline$|\mathrm{L}-\mathrm{NS}|$ & $(0.60,1.31)$ & $(0.28,1.15)$ & $(0.09,1.09)$ \\
\hline$|L-E W|$ & $(0.18,0.02)$ & $(0.04,0.11)$ & $(0.57,0.72)$ \\
\hline \multirow[t]{3}{*}{ Average sigma (K) } & 21.00218 & 17.52931 & 38.39434 \\
\hline & \multicolumn{3}{|l|}{ FP04 } \\
\hline & $2011 / 12 / 6$ & $2012 / 11 / 23$ & $2012 / 1 / 3$ \\
\hline$|N-S|$ & $(0.08,0.11)$ & $(0.03,0.04)$ & $(0.03,0.08)$ \\
\hline$|E-W|$ & $(0.04,0.07)$ & $(0.07,0.07)$ & $(0.03,0.01)$ \\
\hline$|N S-E W|$ & $(3.75,6.22)$ & $(3.74,6.28)$ & $(3.65,6.06)$ \\
\hline$|\mathrm{L}-\mathrm{NS}|$ & $(5.00,7.59)$ & $(4.61,6.99)$ & $(5.03,7.41)$ \\
\hline$|L-E W|$ & $(1.25,1.27)$ & $(0.88,0.71)$ & $(1.38,1.36)$ \\
\hline Average sigma (K) & 22.06626 & 24.60057 & 11.01532 \\
\hline
\end{tabular}

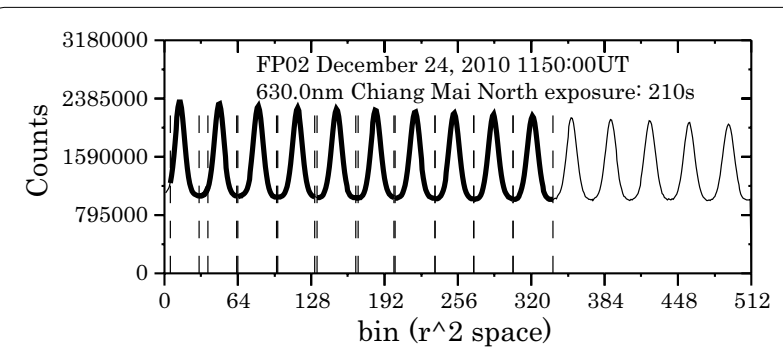

Fig. 7 Example of azimuthally integrated fringes (thin lines). This was obtained by FP02 for 630.0 nm (sky) on December 24, 2010, at Chiang Mai, Thailand after the correction of the center of the interference fringe. Thick lines indicate the fitting of Eq. (1). The thin vertical dashed lines indicate the intervals of the free spectral ranges used for the fitting conclude that the distortion of the optics due to the scanning head weight caused the unrealistic temperatures in Fig. 6, and that we could fix this problem by considering the fringe center shift for each scanning direction. We compared the wind velocities obtained with and without the correction of the center location, and did not find any discernible differences of the two winds. This fact indicates that the determination of the center location does not much affect the fringe peak locations, though it significantly affects the fringe widths.

We believe that this distortion of the optics does not affect the parallelism of the two etalon plates, because the etalon plates are not directly connected to the etalon mount, and the etalon cell is a shield cell to avoid effects 


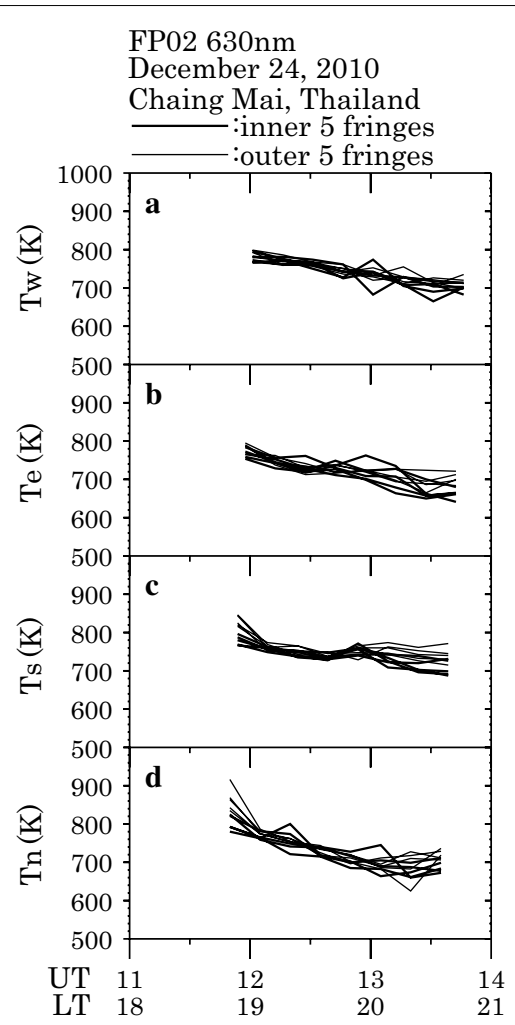

Fig. 8 Temperatures for $\mathbf{a}$ west, $\mathbf{b}$ east, $\mathbf{c}$ south and $\mathbf{d}$ north. This was measured by FP02 for 630.0-nm airglow at Chiang Mai on December 24, 2010 after the correction of the center of the interference fringe

of the atmospheric pressure variation. The etalon cell is mounted in the optics using adjustable screws from the bottom, and thus, the weight of the sky scanner head does not come to the etalon itself.

We also checked whether or not the shift of the fringe center affects the estimation of wind velocities by Shiokawa et al. (2012). Figure 9 shows a comparison of wind velocities obtained with and without the fringe center corrections. Figure $9 \mathrm{a}, \mathrm{b}$, and thin lines in (e) and (f) are obtained without the fringe center correction using the Gaussian fitting described by Shiokawa et al. (2012). Figure 9c, d, and thick lines in (e, f) are obtained with the fringe center correction using the Fourier fitting described in this paper. We can see that the difference of wind velocities with and without the center correction is very small within the statistical error. This is because the wind velocity is determined from the shift of fringe peak locations which is less affected by the center location and azimuthal integration, while the temperature is determined by the width of the fringes, which are directly affected by the center location through the azimuthal fitting. We also found that the wind estimated by Fourier fitting provides less statistical error than those estimated by the Gaussian fitting.

\section{Statistical analysis}

Figure $10 \mathrm{a}-\mathrm{d}$ shows all temperatures (crosses) obtained from $630.0-\mathrm{nm}$ airglow for every $3.5 \mathrm{~min}$ by the four FPIs (FP01-FP04) for 3-5 years. The interval of data set is shown in each panel, which covers different seasonal periods. Most temperatures are from 600 to $1000 \mathrm{~K}$ and decrease from evening to morning for Chiang Mai, Kototabang and Darwin. This is because of cooling of the thermosphere at night. The temperatures tend to be stable for Troms $\varnothing$ at auroral zone. Unrealistic low temperatures below $400 \mathrm{~K}$ are seen in (b)-(d).

Figure 11a-d (left panels) shows averaged temperatures (solid lines) and number of data (dashed lines) obtained at the same sites and for the same interval as Fig. 10. We also show the curves obtained by averaging the temperatures of MSIS-90E (Hedin 1991) and GAIA (Jin et al. 2011) averaged over the same months to those of the actual observation data. We took nighttime variations of these model temperatures for the first day of all the months when the actual data were available. The temperatures obtained by FPIs and MSIS-E90 and GAIA decrease from evening to morning. However, temperatures obtained by FPIs in (a)-(d) are about $200 \mathrm{~K}$ lower than that obtained by MSIS-E90 and GAIA. This is probably because of the unrealistic low temperatures below $400 \mathrm{~K}$ shown in Fig. 10.

\section{Discussion}

Figure 12 shows relations between temperatures and peak counts per one CCD pixel at the same site and for the same interval of Fig. 10. We found that when temperatures are below $400 \mathrm{~K}$, peak counts (per pixel) are very low. The dashed line indicates 257 counts per pixel which is three times of the readout noise of the CCD camera. Since the dark noise of the CCD camera is negligible compared with the readout noise, we use this value (three times of the readout noise) as a threshold to cut out the low-count data. When the peak count is roughly higher than this value, we found that the output temperature is not extremely small.

By considering this result in Fig. 12, we remade the statistical analysis without the data for which peak count of interference fringe is below 257 counts. The results are shown in Figs. 10e-h and 11e-h. In Fig. 10e-h, there is no temperature below $400 \mathrm{~K}$. In Fig. 11e-h, temperatures obtained by FPIs are comparable to temperatures obtained by MSIS-E90 and GAIA. Better fitting is obtained for the temperatures at Chiang Mai (Fig. 11f) and Darwin (Fig. 11h) for the GAIA model than the MSIS-90E. On the other hand, the GAIA temperature at Tromsø in Fig. 11e is about $200 \mathrm{~K}$ lower than that of FPI and MSIS-90E. This may be because the current GAIA model does not contain thermospheric heating due to 


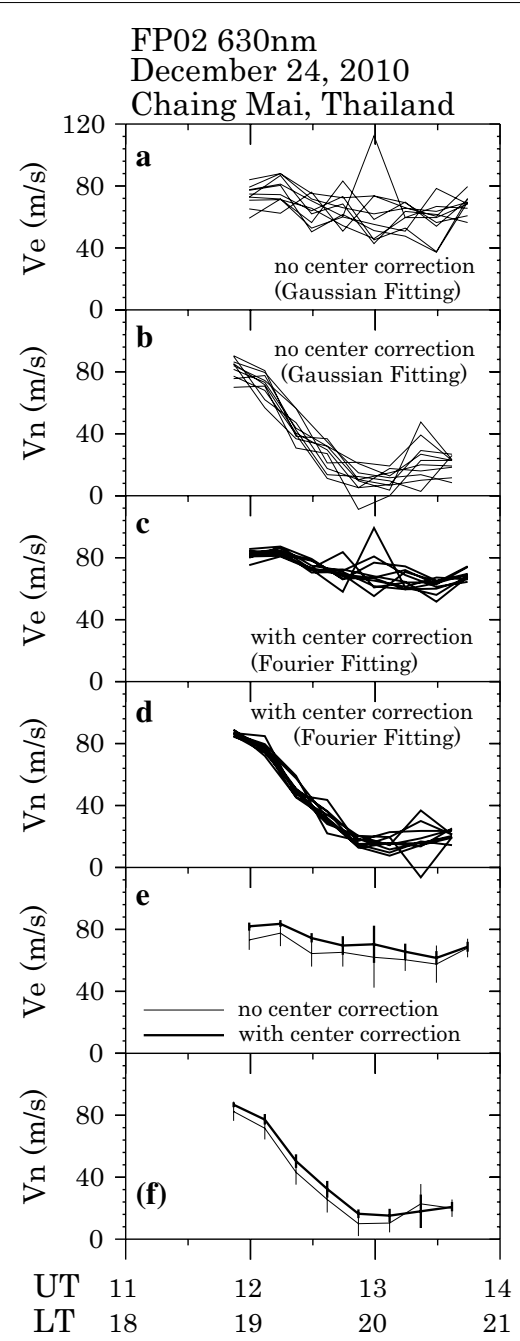

Fig. 9 Wind velocities obtained without the fringe center correction for $\mathbf{a}$ eastward, $\mathbf{b}$ northward, with the fringe center correction for $\mathbf{c}$ eastward and $\mathbf{d}$ northward, and their comparison in e eastward and f northward. These wind velocities were measured by FP02 for 630.0nm airglow at Chiang Mai on December 24, 2010

auroral energy input at high latitudes. The difference of GAIA and FPI temperatures at Kototabang (Fig. 11g) at around local midnight is possibly due to ambiguity of the location of midnight temperature maximum (MTM) in the model, because Kototabang is located at geographic equator at $0.2 \mathrm{~S}$, which is the main region where the MTM is occurring.

We also have tried cutoff threshold values smaller than the 257 counts, but the statistical comparison with the MSIS and GAIA model shows that the observed temperature is smaller than these models. Thus, we decided to use this threshold to remove the low-intensity data.

Figure 13 shows an example of azimuthally integrated fringes (thin lines) for which extremely low temperatures were obtained. The average temperature obtained by fitting (1) to these eight fringes is $154 \mathrm{~K}$ with a standard deviation of $45 \mathrm{~K}$. From this figure, we found that not only peak count is very low, but also the fringe shape is rather distorted, forming a shoulder-like shape. Thus, we could not fit Eq. (1) to the whole one free spectral range of the fringes. This distorted fringe shape may be caused by the emission of $\mathrm{OH}(9-3)\left(K^{\prime \prime}=3\right), P_{1}, P_{2},(630.68$, $629.87 \mathrm{~nm}$ ) which are within the pass band of the interference filter (FWHM $=2.5 \mathrm{~nm}$ ) of the FPIs. When the 630.0 -nm airglow intensity is very low, these $\mathrm{OH}$ emissions would become non-negligible. This can cause underestimation of the fringe width, and thus extremely low temperatures are obtained.

In order to investigate this possibility of the contamination of $\mathrm{OH}$ emissions, we made a model calculation on the overlap of the $\mathrm{OI}$ and $\mathrm{OH}$ lines in Fig. 14. This figure indicates the interference fringe patterns with etalon gaps of (a) $15 \mathrm{~mm}$ (FSR: $0.013 \mathrm{~nm}$ ) and (b) $20 \mathrm{~mm}$ (FSR: $0.0098 \mathrm{~nm})$ with the input of the OI $(630.0 \mathrm{~nm})$ line and $\mathrm{OH}(630.68,629.87 \mathrm{~nm})$ lines in the same intensity with a zero temperature. The reflectivity $R=0.76$ of FP02-FP04 is used, corresponding to a finesse of 11.4. As shown in Fig. 14a, the fringe peak of the OI 630.038-nm line completely overlaps with the fringe peak of $\mathrm{OH}\left(9-3, K^{\prime \prime}=3\right.$, $P_{1}, 630.68 \mathrm{~nm}$ ) line (dashed curve), while the fringes of $\mathrm{OH}\left(9-3, K^{\prime \prime}=3, P_{2}, 629.87 \mathrm{~nm}\right)$ appear at slightly different location. This configuration occurs for the etalon gap of $15 \mathrm{~mm}$ which we used for our FPIs. If the etalon gap becomes $20 \mathrm{~mm}$, the three lines show fringe peaks at different locations, as shown in Fig. 14b. The $\mathrm{OH}$ emission should give much lower temperature, since it is coming from the mesopause region where the temperature is $\sim 200 \mathrm{~K}$. Since $\mathrm{OH}\left(P_{2}, 629.87 \mathrm{~nm}\right)$ is at higher state than $\mathrm{OH}\left(P_{1}, 630.68 \mathrm{~nm}\right)$, we should have larger intensity in the $\mathrm{OH}\left(P_{1}, 630.68 \mathrm{~nm}\right)$ line. Thus, we conclude that the low temperatures obtained by our FPIs for low airglow intensities are probably due to the contamination of $\mathrm{OH}$ lines, particularly due to the overlapping of the $\mathrm{OH}$ $(630.68 \mathrm{~nm})$ fringes on the OI $(630.038 \mathrm{~nm})$ fringes.

This problem may be avoided if one uses the etalon gap different from $15 \mathrm{~mm}$, as shown in Fig. 14b. However, there is still a difficulty to distinguish the fringes caused by $\mathrm{OH}$ lines from the $\mathrm{OI}(630.0 \mathrm{~nm})$ fringes. If one uses narrower band-pass filter on the top of the FPI, this problem would be avoided. However, narrower band-pass filter reduces the transmission, giving less sensitivity of the FPI.

As shown in Fig. 12, the temperature of FP01 never goes below $400 \mathrm{~K}$, while temperatures of FP02-04 go below $400 \mathrm{~K}$. This means that even for very small airglow intensity the FP01 does not produce temperatures below $400 \mathrm{~K}$. Thus, this difference would not be because of the difference of airglow intensities at high and low latitudes. 


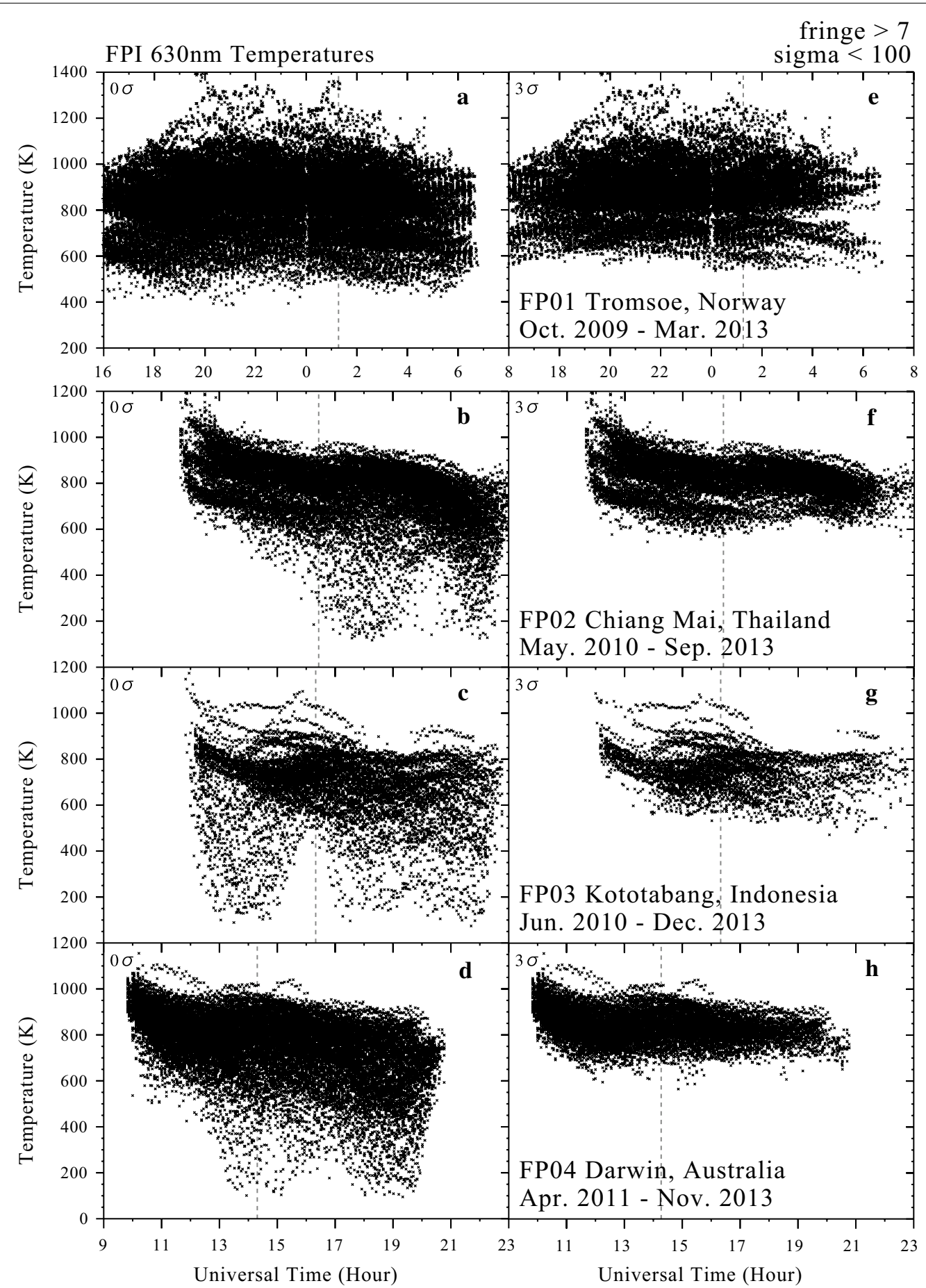

Fig. 10 Relation between temperatures (crosses) obtained by FPIs and universal time. This was obtained at a, e Tromsø for October 11, 2009-May 24, 2013, b, f Chiang Mai for May 2, 2010-September 14, 2013, c, g Kototabang for June 11, 2010-December 31, 2013 and d, h Darwin for April 6, 2011-November 3, 2013. Left panels (a-d) and right (e-h) panels indicate original temperatures and the temperatures without the data when the airglow intensity is very low, respectively. Dashed lines indicate local midnight

As shown by the model calculation in Fig. 14, the location of $\mathrm{OH}$ fringe peak depends only on the etalon gap. Both FP01 and FP02-04 have the same etalon gap of
$15 \mathrm{~mm}$. The interference filters of both FP01 and FP02-04 have same specifications of FWHM $=2.5 \mathrm{~nm}$. The focal lengths of the imaging lens just above the CCD camera 

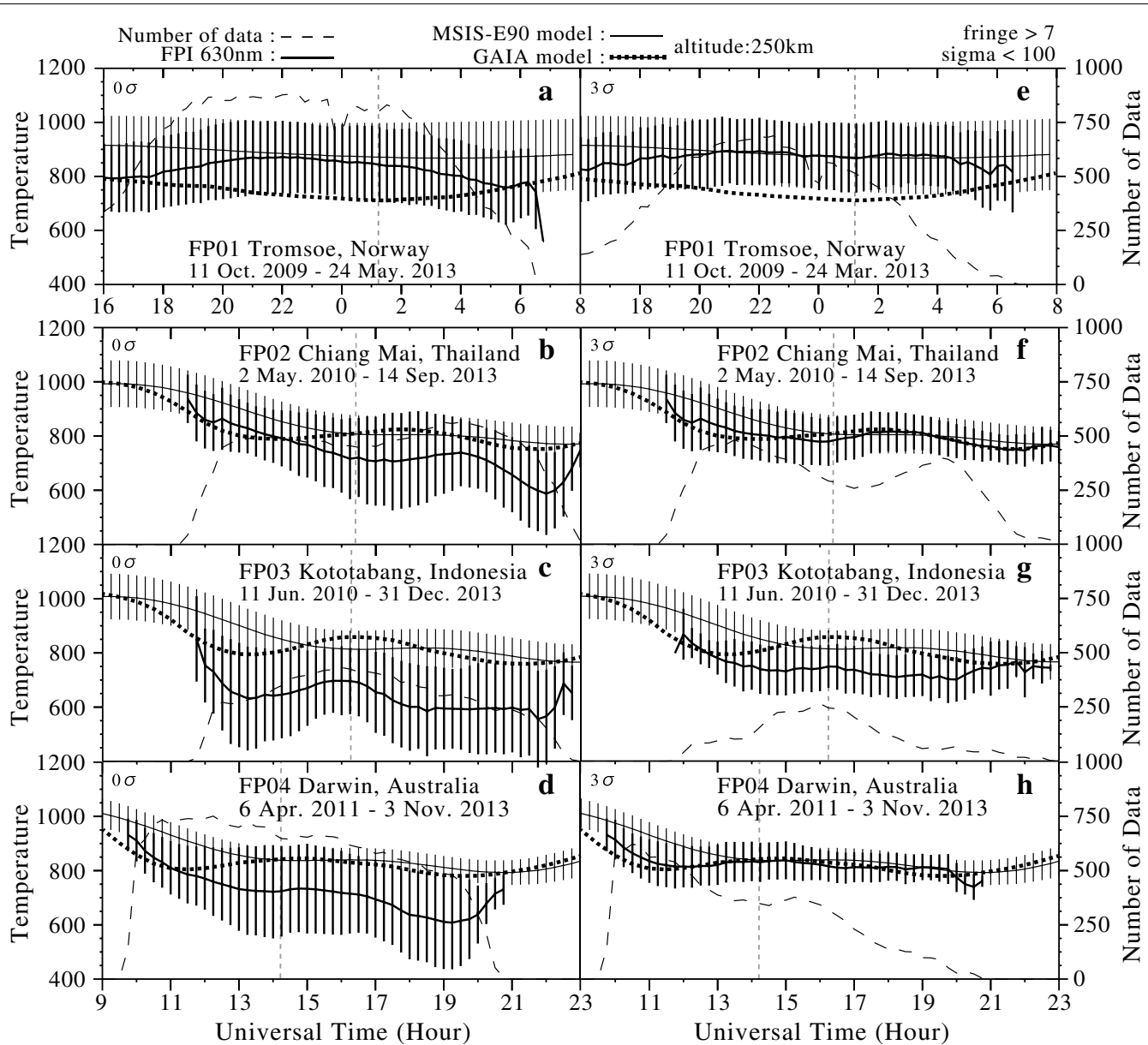

Fig. 11 Averaged temperatures (solid lines) and number of data (dashed lines). This was obtained at $\mathbf{a}, \mathbf{e}$ Troms $\varnothing, \mathbf{b}$, f Chiang Mai, $\mathbf{c}, \mathbf{g}$ Kototabang and $\mathbf{d}, \mathbf{h}$ Darwin for the same interval of Fig. 10. Thick lines show temperatures averaged for every 15 min obtained by FP01-04. Thin lines and dotted lines show that obtained by the MSIS-E90 model and the GAIA model, respectively, at an altitude of $250 \mathrm{~km}$. The error bars indicate standard deviations of the whole data. Left panels $(\mathbf{a}-\mathbf{d})$ and right panels $(\mathbf{e}-\mathbf{h})$ indicate original data and the data that removed the data obtained when the airglow intensity is very low, respectively

are $270 \mathrm{~mm}$ for FP02-FP04 and $300 \mathrm{~mm}$ for FP01. The other difference is the finesse of the etalon, where FP01 has larger finesse with higher reflectivity. Because the FPI temperature is essentially measured from the width of the fringes, the larger finesse allows more accurate measurements of the temperature, and may contribute to differentiate the $\mathrm{OH}$ contamination. The smaller finesse was chosen for the small-etalon FPIs of FP02-04, in order to increase the etalon transmission, because the small-etalon FPIs have less sensitivity due to the small-aperture size.

We also note that the etalon gap drift by variation of ambient temperature of the FPI may affect the accuracy of the FPI temperature measurement through widening the fringe width during the drift. As shown by Shiokawa et al. (2012), we can monitor the drift of the etalon gap as equivalent wind velocity, which can be $100 \mathrm{~m} / \mathrm{s} / \mathrm{h}$ in significant case. Since the exposure time of the FPI is $210 \mathrm{~s}$, the etalon gap drift can be $6 \mathrm{~m} / \mathrm{s}$ in equivalent wind velocity during one exposure. This is about $0.1 \%$ of the free spectral range of our FPI $(6300 \mathrm{~m} / \mathrm{s}$ in equivalent wind velocity).

\section{Conclusions}

In this paper we evaluate thermospheric temperatures obtained by four new FPIs operated by STEL/ ISEE, Nagoya University. Three of them used small 70-mm-diameter etalons. In the preliminary analysis, we had the following problems.

1. Large differences of temperatures were obtained from different fringes. 


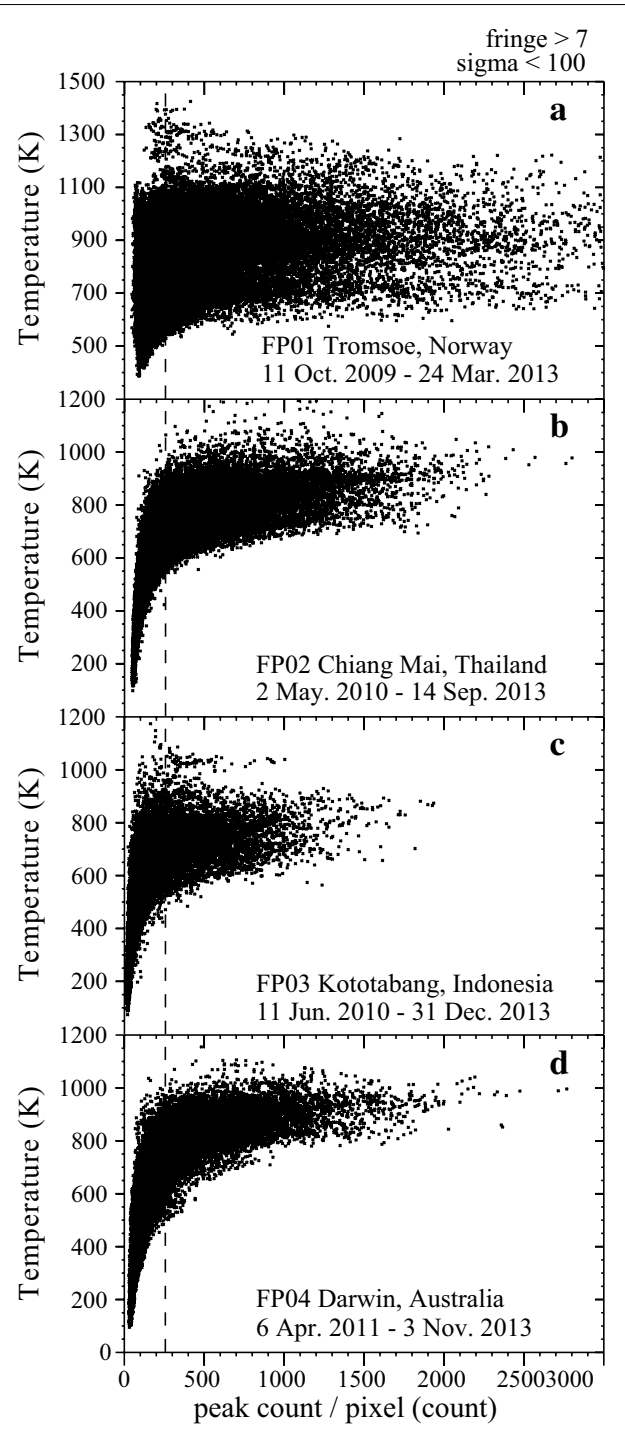

Fig. 12 Relation of temperature and peak count (per pixel) estimated from interference fringes. This was obtained at $\mathbf{a}, \mathbf{e} T r o m s \varnothing$,

b, f Chiang Mai, c, $\mathbf{g}$ Kototabang and $\mathbf{d}, \mathbf{h}$ Darwin for the same interval of Fig. 10. Dashed line shows 257 counts per pixel which is three times of the readout noise of the CCD camera

2. The temporal temperature variations were different for east-west and south-north directions.

In the above preliminary study, we had used the fringe center calculated by averaging for all ring patterns collected in a night. The spectral widths of the sky fringes are proportional to the width of the fringes. Thus, the center determination is very important for temperature estimation. We found that fringe centers for four directions of the sky are different, probably because of distortion of the

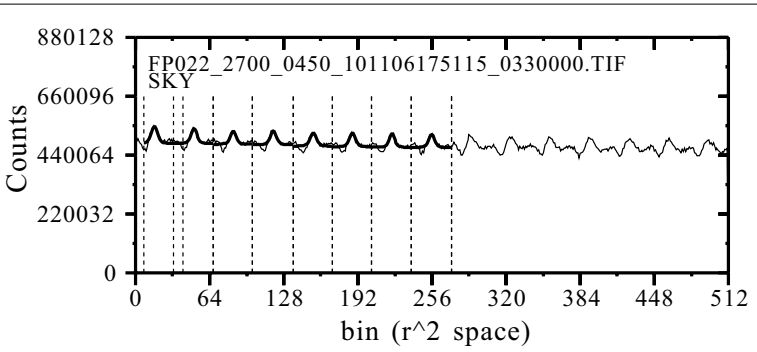

Fig. 13 An example of azimuthally integrated fringes (thin lines). This was obtained by FP02 for 630.0 nm (sky) on November 6, 2010, at Chiang Mai, Thailand, for which extremely low temperatures were obtained. Thick lines indicate the fitting of Eq. (1). The thin vertical dashed lines indicate the intervals of the free spectral ranges used for the fitting

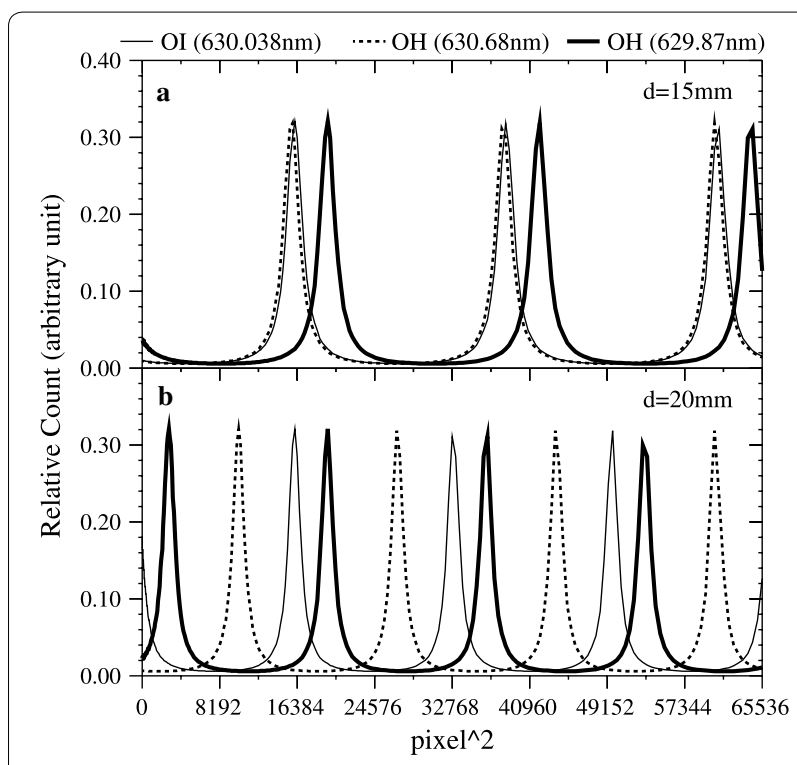

Fig. 14 Interference fringes calculated for the three airglow emissions at $\mathrm{Ol} 630.038 \mathrm{~nm}, \mathrm{OH} 630.68 \mathrm{~nm}$ and $\mathrm{OH} 629.87 \mathrm{~nm}$ with etalon gaps of $\mathbf{a} 15 \mathrm{~mm}$ and $\mathbf{b} 20 \mathrm{~mm}$

optics due to the change in the balance of the scanning head weight depending upon the azimuthal direction chosen for each of the three small FPIs. To fix this problem, we determined the fringe center for each direction. Then, we could determine reliable temperatures with an accuracy of about $10-40 \mathrm{~K}$ which is estimated as the standard deviation of ten fringe temperatures. We also confirmed that the estimation of the wind velocity is not affected by this distortion of the optics.

Next, we performed a statistical analysis of temperatures obtained by this new method of center determination and obtained the following results. 
1. Temperatures decrease from evening to morning at low latitudes, because of cooling of the thermosphere at night.

2. Temperatures obtained by the MSIS-E90 and GAIA models have similar trend.

However, we noticed that extremely low temperatures below $400 \mathrm{~K}$ were obtained when airglow intensity is very low.

We derived the relation between peak counts and temperatures obtained from interference fringes. We decided to remove the data which have peak count less than 257 counts (three times of the readout noise of the CCD camera). Then we obtained the following results.

3. Temperatures below $400 \mathrm{~K}$ were removed.

4. Average temperatures obtained by FPIs became comparable to that of the MSIS-E90 empirical model and the GAIA simulation model. Better fitting was obtained for the GAIA model at low latitude stations.

Using a model calculation of FPI fringes, we conclude that the contamination of emissions from $\mathrm{OH}(9-3)$ $\left(\mathrm{K}^{\prime \prime}=3\right), P_{1}$ at the wavelength of $630.68 \mathrm{~nm}$ caused the unrealistically low values of temperature when the 630.0$\mathrm{nm}$ airglow intensity is very low.

\section{Abbreviation \\ FPI: Fabry-Perot interferometer.}

\section{Authors' contributions}

YN improved the analysis procedures for calculating the thermospheric temperatures, which was originally made by KS, for small Fabry-Perot interferometers. He also carried out the statistical analysis for FP01-04 and wrote the manuscript. KS initiated the study as the Principal Investigator of the OMTIs project, developed the Fourier fitting software to calculate the temperature, made the model calculation of Fig. 14, improved the manuscript including interpretation as the supervisor of $\mathrm{YN}$ and took a lead to revise the manuscript after the YN's graduation of university on March 2015. YO helped to carry out the operation of the four FPIs. SO and SN carried out the operations of the FPI at Tromsø, Norway, and MK, TK and SK managed the operations of the FPI at Chiang Mai. CYY and DN/CY managed the operation of the FPIs at Kototabang and Darwin, respectively. JM advised the procedure of the temperature calculation and edited the manuscript. HS and HJ provided the GAIA model temperature for comparison with the FPI data. All authors read and approved the final manuscript.

\footnotetext{
Author details

1 Solar-Terrestrial Environment Laboratory (STEL), Nagoya University, Chikusa-ku, Nagoya 464-8601, Japan. ${ }^{2}$ Present Address: Toyota Co. Ltd, Toyota, Japan. ${ }^{3}$ Institute for Space-Earth Environmental Research (ISEE), Nagoya University, Chikusa-ku, Nagoya 464-8601, Japan. ${ }^{4}$ Chiang Mai University, Chiang Mai 50200, Thailand. ${ }^{5}$ Ionospheric Prediction Service (IPS), Bureau of Meteorology - Space Weather Services, Level 15, 300 Elizabeth St., Surry Hills, NSW 2000, Australia. ${ }^{6}$ Clemson University, Clemson, SC 29634-0978, USA. ${ }^{7}$ National Institute of Information and Communications Technology, Koganei 184-8795, Japan.
}

\section{Acknowledgements}

We appreciate T. Kato, M. Sato, Y. Kato, Y. Hamaguchi and Y. Yamamoto of the Solar-Terrestrial Environment Laboratory/ Institute for Space-Earth Environmental Research (ISEE), Nagoya University, for their skillful support in developing the FPIs. The FPI observation at Chiang Mai was carried out as a part of an international cooperative research project among Chiang Mai University, Thailand, National Institute of Information and Communications Technology, Japan. We thank Mike Kosch of University of Lancaster allowing us to operate the FP01 in his hut. This work was supported by JSPS Grants-in-Aid for Scientific Research (20244080, 15H05815 and 16H06286), the JSPS Coreto-Core Program, B. Asia-Africa Science Platforms, by the Special Funds for Education and Research (Energy Transport Processes in Geospace) and by the IUGONET Project from MEXT, Japan.

\section{Competing interests}

The authors declare that they have no competing interests.

\section{Publisher's Note}

Springer Nature remains neutral with regard to jurisdictional claims in published maps and institutional affiliations.

Received: 12 September 2016 Accepted: 12 April 2017

Published online: 24 April 2017

\section{References}

Biondi MA, Sipler DP, Zipf ME, Baumgardner JL (1995) All-sky Doppler interferometer for thermospheric dynamics studies. Appl Opt 34:1646-1654. doi:10.1364/AO.34.001646

Hedin AE (1991) Extension of the MSIS thermosphere model into the middle and lower atmosphere. J Geophys Res 96:1159. doi:10.1029/90JA02125

Hernandez G, Roble RG (1995) Thermospheric nighttime neutral temperature and winds over Fritz Peak Observatory: observed and calculated solar cycle variation. J Geophys Res 100:14647. doi:10.1029/95JA00565

Jin H, Miyoshi Y, Fujiwara H, Shinagawa H, Terada K, Terada N, Ishii M, Otsuka Y, Saito A (2011) Vertical connection from the tropospheric activities to the ionospheric longitudinal structure simulated by a new Earth's whole atmosphere-ionosphere coupled model. J Geophys Res 116:A01316. doi: 10.1029/2010JA015925

Killeen TL, Hays PB (1984) Doppler line profile analysis for a multichannel Fabry-Perot interferometer. Appl Opt 23:612. doi:10.1364/AO.23.000612

Killeen TL, Won Y-I, Niciejewski RJ, Burns AG (1995) Upper thermosphere winds and temperatures in the geomagnetic polar cap: solar cycle, geomagnetic activity, and interplanetary magnetic field dependencies. J Geophys Res 100:21327. doi:10.1029/95JA01208

Makela JJ, Meriwether JW, Lima JP, Miller ES, Armstrong SJ (2009) The remote equatorial nighttime observatory of ionospheric regions project and the international heliospherical year. Earth Moon Planets 104:211-226. doi:10.1007/s11038-008-9289-0

Makela JJ, Meriwether JW, Huang Y, Sherwood PJ (2011) Simulation and analysis of a multi-order imaging Fabry-Perot interferometer for the study of thermospheric winds and temperatures. Appl Opt 50:4403-4416. doi:10.1364/AO.50.004403

Meriwether JW, Mirick JL, Biondi MA, Herrero FA, Fesen CO (1996) Evidence for orographic wave heating in the equatorial thermosphere at solar maximum. Geophys Res Lett 23:2177-2180. doi:10.1029/96GL01976

Meriwether JW, Biondi MA, Herrero FA, Fesen CG, Hallenback DC (1997) Optical interferometric studies of the nighttime equatorial thermosphere: enhanced temperatures and zonal wind gradients. J Geophys Res 102:20041. doi:10.1029/97JA01463

Meriwether JW, Makela JJ, Huang Y, Fisher DJ, Buriti RA, Medeiros AF, Takahashi $H$ (2011) Climatology of the nighttime equatorial thermospheric winds and temperatures over Brazil near solar minimum. J Geophys Res 116:A04322. doi:10.1029/2011JA016477 
Shiokawa K, Katoh Y, Satoh M, Ejiri MK, Ogawa T, Nakamura T, Tsuda T, Wiens RH (1999) Development of optical mesosphere thermosphere imagers (OMTI). Earth Planets Space 51:887-896. doi:10.1186/BF03353247

Shiokawa K, Kadota T, Otsuka Y, Ogawa T, Nakamura T, Fukao S (2003) A twochannel Fabry-Perot interferometer with thermoelectric-cooled CCD detectors for neutral wind measurement in the upper atmosphere. Earth Planets Space 55:271-275. doi:10.1186/BF03351759

Shiokawa K, Otsuka Y, Ogawa T (2009) Propagation characteristics of nighttime mesospheric and thermospheric waves observed by optical mesosphere thermosphere imagers at middle and low latitudes. Earth Planets Space 61:479-491. doi:10.1186/BF03353165

Shiokawa K, Otsuka Y, Oyama S, Nozawa S, Satoh M, Katoh Y, Hamaguchi Y, Yamamoto Y, Meriwether J (2012) Development of low-cost sky-scanning Fabry-Perot interferometers for airglow and auroral studies. Earth Planets Space 64:1033-1046. doi:10.5047/eps.2012.05.004

Tohmatsu T (1973) Compendium of aeronomy. Terra Publishing, Tokyo

\section{Submit your manuscript to a SpringerOpen ${ }^{\odot}$ journal and benefit from:}

- Convenient online submission

- Rigorous peer review

- Immediate publication on acceptance

- Open access: articles freely available online

- High visibility within the field

- Retaining the copyright to your article 